\title{
Low Threshold, Single-mode Laser Based on Individual CdS Nanoribbons in Dielectric DBR Microcavity
}

Qinglin Zhang, ${ }^{1 \ddagger}$ Shao-Wei Wang, ${ }^{2 \ddagger}$ Xingxing Liu, ${ }^{2}$ Tianren Chen, ${ }^{1}$ Huafen Li, ${ }^{2}$ Junwu Liang, ${ }^{1}$ Weihao Zheng, ${ }^{1}$ Ritesh Agarwal, ${ }^{3}$ Wei $\mathrm{Lu}^{2} *$ and Anlian Pan ${ }^{1} *$

${ }^{1}$ Key Laboratory for Micro-Nano Physics and Technology of Hunan Province, Key Laboratory for Micro/Nano Optoelectronic Devices of Ministry of Education, School of Physics and electronics, Hunan University, Changsha 410082, China

${ }^{2}$ National Laboratory for Infrared Physics, Shanghai Institute of Technical Physics, Chinese Academy of Sciences, Shanghai 200083, China

${ }^{3}$ Department of Materials Science and Engineering, University of Pennsylvania, Philadelphia, Pennsylvania 19104, United States

$\$$ The authors contributed equally for this paper.

*Corresponding Author: E-mail: (anlian.pan@ @nu.edu.cn for Anlian Pan; luwei@mail.sitp.ac.cn for Wei Lu)

\begin{abstract}
Single-mode lasers with low threshold are attractive for their potential applications in many areas, such as optical communication, signal processing and displays. Here we report a nanoscale single-mode laser with CdS nanoribbons (NRs) sandwiched between two dielectric distributed Bragg reflectors (DBRs). Under optical pumping, the band edge emission of CdS ribbons can be effectively confined and give lasing in the DBR microcavity, with the lasing threshold as low as $\sim 8 \mu \mathrm{J} / \mathrm{cm}^{2}$, which is one order lower than those of bare CdS ribbons for comparison. More importantly, the CdS-DBR laser can realize single-mode emission, for the length of the resonance cavity can support a mode spacing larger than the bandwidth of the
\end{abstract}


optical gain. Light polarization measurements demonstrate the single-mode lasing has a polarization degree as high as 97.4\%. Time-resolved photoluminescence (TRPL) measurements further reveal that the lasing action comes from the electron-hole plasma recombination process. Moreover, the wavelength of the lasing mode can be broadly tuned by changing the thickness of CdS NRs. This low-threshold single-mode laser may find applications in highly integrated photonics devices and systems.

Keywords: Single-mode laser, Dielectric DBR, Microcavity, Nanoribbons

\section{Introduction}

Miniaturized coherent lasers have been attracting growing attentions, due to their potential wide applications including optical communications, signal processing, high throughput sensing, and highly integrated photonic systems [1-7]. Low dimensional semiconductor nanowires (NWs) or nanoribbons (NRs) are considered as the most important and promising candidates for the realization of nanoscale lasers and amplifiers, because they can act as not only the gain media but also the resonance cavities for light amplification [8-13].

Generally, the light guided in the semiconductor NWs or NRs will be amplified when the round trip loss can be compensated by the round-trip gain supported by the feedback of endface reflection. However, the round trip loss is usually very large, due to the low reflectivity at the endfaces as well as the large transmission losses resulted from surface scattering and selfabsorption, which results in high lasing threshold of nanostructured lasers [14-17]. Several strategies have been proposed to lower the lasing threshold through reducing the round trip loss. To decrease the scattering losses, metal surface plasmon has been used to localize the scattering light at the surface of $\mathrm{CdS}$ [18] and GaN [19] nanostructured cavities, so that their lasing threshold can be respectively lowered significantly [20]. A. Das et al. [21] have realized a ultralow threshold polariton lasing from a single GaN NW enclosed in a dielectric 
microcavity formed with a top and a bottom distributed Bragg reflectors (DBRs) of $\mathrm{SiO}_{2} / \mathrm{TiO}_{2}$, which causes the cavity field to be mainly confined in the NW to greatly increase the interaction between the excitons of the GaN NW and the cavity modes. Recently, composition-symmetric $\mathrm{CdS}_{\mathrm{x}} \mathrm{Se}_{1-\mathrm{x}} \mathrm{NWs}$ have been used to minimize the self-absorption induced optical loss in nanowire lasers, with the lasing threshold several times decreased compared to the conventional composition homogeneous nanostructures [22].

Furthermore, the resonance occurrence in a NW or NR cavity formed by endface reflection $[4,14,23,24]$ requires that the optical path of the cavity equals to an integer multiple of halfresonance-wavelength to form the standing waves, which usually supports a multimode laser emission. The multimode laser emission can result in broadening of the temporal pulse and the false signaling due to the group velocity dispersion [25], which is undesired in the digitized optical communication and signal processing [26]. This can be greatly avoided by using the single-mode laser, due to its high monochromaticity and stability. One can obtain single-mode semiconductor nanostructured lasers by shortening the length of the lasing cavity to extend the mode-spacing until the spacing is larger than the bandwidth of the optical gain [27-29]. However, reducing the cavity length may result in the dramatic increase of the lasing threshold, due to the decrease of the round-trip gain [30-32]. Therefore, the realization of the low-threshold, single mode lasing is one of the most important issues for the practical application of nanolasers. Although the laterally [33-36] and axially [37] coupling nanowire cavities provided a promising route to realize single-mode lasing with significant reduction of lasing threshold, they involved either difficult micromanipulation or complex fabrication process, which bring disadvantages in their practical applications. Based on the numerical simulation, L. Chen et al. [38] proposed that the single-mode lasing could be obtained through the formation of DBR mirrors at the both ends of a single GaN NW, where the high reflectivity DBRs could reduce the cavity loss and hence the lasing threshold. However, it is 
still a challenge to experimentally realize single mode semiconductor nanostructured lasers with ultralow lasing threshold [39, 40].

In this work, CdS NRs were successfully embedded into a high-quality dielectric DBR cavity (schematically shown in Figure 1a). When the reflection band of the dielectric DBR was adjusted to well match the emission band of the CdS NRs, the emitted light will be reflected repeatedly between the two DBRs (schematically shown in Figure 1b), and can easily reach resonance and amplification to give lasing even under very low optical pumping. The very short length of the resonant cavity at the thickness direction makes the lasing be high-quality single mode, and the mode wavelength can be tuned through changing the thickness of the CdS NR. The presented low-threshold, single-mode lasers may find potential applications in on-chip optical communication, signal process, and integrated photonics.

\section{Experiments}

\subsection{Preparation and structure characterization of CdS NRs}

CdS NRs were synthesized with a chemical vapor deposition (CVD) route as reported previously [41]. SEM characterization shows the as-grown NRs have a smooth surface, with the thickness at the range from several tens to several hundreds of nanometer (see Figure S1).

\subsection{Fabrication of the dielectric DBR structures}

Figure 1a shows schematically the single CdS NR embedded in the dielectric DBR microcavity. The full device was designed with the resonance wavelength $\lambda_{0}=520 \mathrm{~nm}$, and experimentally fabricated as follows. Firstly, $59 \mathrm{~nm}\left(\lambda_{0} / 4 \mathrm{n}_{\mathrm{T}}, \mathrm{n}_{\mathrm{T}}=2.21\right.$, the reflective index of $\left.\mathrm{TiO}_{2}\right) \mathrm{TiO}_{2}$ layer was deposited on a $\mathrm{K} 9$ glass substrate (see Figure 1a), on which the bottom DBR was constructed by alternately depositing seven pairs of $89 \mathrm{~nm}\left(\lambda_{0} / 4 \mathrm{n}_{\mathrm{S}}, \mathrm{n}_{\mathrm{T}}=1.46\right.$, the 
reflective index of $\left.\mathrm{SiO}_{2}\right) \mathrm{SiO}_{2}$ layers and $59 \mathrm{~nm} \mathrm{TiO}$ layers. On top of the bottom $\mathrm{DBR}, 73$ $\mathrm{nm} \mathrm{SiO}_{2}$ was deposited. Then individual $\mathrm{CdS}$ NRs were transferred from the as-grown substrate onto the surface of the as-prepared $\mathrm{TiO}_{2}-\mathrm{DBR}-\mathrm{SiO}_{2}$ using a micro-probe manipulation technique. Finally, a layer of $105 \mathrm{~nm} \mathrm{SiO} 2$ and the top DBR (eight pairs of $\mathrm{TiO}_{2} / \mathrm{SiO}_{2}$ layers with the same thickness as that in bottom DBR) were in turn prepared. Both the bottom and top DBRs have been prepared by Leybold ARES1110 High Vacuum Coating System, $99.99 \%$ purity of $\mathrm{TiO}_{2}$ and $\mathrm{SiO}_{2}$ were used as coating material. Base vacuum of the coating chamber was $2 \times 10^{-4} \mathrm{~Pa}$. Argon (99.999\%) of $30 \mathrm{sccm}$ and oxygen (99.999\%) of 35 sccm were used as working gases when $\mathrm{TiO}_{2}$ was evaporated, while only argon of $30 \mathrm{sccm}$ was used when $\mathrm{SiO}_{2}$ was evaporated. The chamber temperature was $150^{\circ} \mathrm{C}$ during the coating process. The evaporation rates were kept at $0.5 \mathrm{~nm} / \mathrm{s}$ and $1.0 \mathrm{~nm} / \mathrm{s}$ for $\mathrm{TiO}_{2}$ and $\mathrm{SiO}_{2}$, respectively. Their thicknesses were precisely controlled by direct optical monitor of OMS5000. The detailed fabrication process can be found in the ref [42]. The length of the cavity includes the thickness of $\mathrm{CdS} \mathrm{NR}\left(\mathrm{d}_{\mathrm{CdS}}\right)$, and the thickness of its adjacent $\mathrm{SiO}_{2}$ layers $\left(d_{S}=73+105=178 \mathrm{~nm}\right)$ (see Figure $\left.1 \mathrm{~b}\right)$. To vary the lasing wavelength, NRs with different thickness, determined with atomic force microscope (AFM) equipped on the confocal microscopy (WITec, alpha-300), were embedded in the same DBR microcavity.

\subsection{Optical measurements of the devices}

The transmission spectrum of the bottom dielectric DBR was measured with the Uv-Vis Spectrophotometer (TU-1901, Beijing Pursee Inc.). The measurments of $\mu-\mathrm{R}$ spectra were conducted on the confocal microscopy (WITec, alpha-300) with reflection mode under the illumination of a white light source [43]. The device was excited by a focused Ti:Sapphire laser at $450 \mathrm{~nm}(100 \mathrm{fs}, 1 \mathrm{kHz}$, Spectra Physics) incident from the bottom substrate with a transmissivity of $\sim 80 \%$, and the emission was collected by a confocal microscope (WITec, 
alpha-300) with a 100× objective (Nikon, NA:0.9) from the top DBR side (see Figure 1a and Figure S2), and then detected by a CCD spectrometer (600 g/mm grating) and a streak camera (Hasmamatsu Universal, C10910) for photoluminescence (PL) and time-resolved PL (TRPL) measurements, respectively. The high spatial resolution of the confocal microscopy makes it possible to measure the $\mu-\mathrm{R}$ spectra and PL spectra at the same region. All of the measurements were conducted at room temperature.

\section{Results and discussion}

Figure 1c gives the large view of some representative $\mathrm{CdS}$ ribbons embedded in the dielectric DBR cavity with one of them broken together with the cavity, at the viewing angle of $45^{\circ}$. Besides the morphology of the ribbons can be clearly observed, the cross sections of one CdS ribbon and both the DBRs can be distinguished obviously, where the CdS ribbon was sandwiched between two DBRs with alternately layered structure (details shown in the inset of Figure 1c). The transmission spectrum of the bottom dielectric DBR (the upper panel of Figure 1d) shows that the reflection band of the reflector is located from 475 to $590 \mathrm{~nm}$ with the reflectivity of $\sim 98.5 \%$ (transmission of $1.5 \%$ ), which matches well with the bandedge emission of bare CdS NRs centered at $508 \mathrm{~nm}$ with a full width at half-maximum (FWHM) of $\sim 10 \mathrm{~nm}$ (lower panel of Figure1d). The inset of Figure 1d gives the microreflectance $(\mu-\mathrm{R})$ spectrum of a representative CdS NRs (with the thickness of $\sim 316 \mathrm{~nm}$ determined by AFM, see Figure S3e in the Supporting Information) sandwiched in the DBRs, which reveals a narrow passband centered at the wavelength of $\sim 520 \mathrm{~nm}$ with the FWHM of $\sim 1.6 \mathrm{~nm}$, corresponding to the cavity mode of the full device [42].

Figure 2a shows the pumping power dependent emission from the same region as the $\mu-\mathrm{R}$ spectrum measurement for the representative CdS NR in the DBR cavity. At low pumping fluence of $4.6 \mu \mathrm{J} / \mathrm{cm}^{2}$, only weak broad emission band from 490 to $523 \mathrm{~nm}$ was observed, 
corresponding to the spontaneous emission (SPE) of CdS [44]. When the exciting fluence was increased to $8.6 \mu \mathrm{J} / \mathrm{cm}^{2}$, a sharp emission line appeared at $\sim 520 \mathrm{~nm}$, superimposed on the weak broad emission band. Further increasing the fluence caused a rapid intensity increase of the sharp line, which finally dominated the spectrum with the FWHM of $\sim 0.5 \mathrm{~nm}$ (quality factor of 1040) at the fluence of $14.6 \mu \mathrm{J} / \mathrm{cm}^{2}$, indicating the occurrence of stimulated emission. The good consistence of the wavelength of the sharp line with the cavity mode (inset of Figure $1 \mathrm{~b}$ ) indicates that the lasing is resulted from the coupling between the CdS NR and the dielectric DBR cavity.

For semiconductor NRs, optical resonance and amplification can easily realize at both the length and the width directions via the endface reflection of the confined light. Light resonance is usually hard to be observed at the thickness direction, due to the large optical loss and limited gain length $[45,46]$. However, when CdS NRs are sandwiched between the top and the bottom dielectric DBRs, the emission light can be well confined in the microcavity defined by the high reflectivity dielectric DBRs, which increases the light gain and facilitates the light resonance along the thickness of the ribbons (schematically see Figure 1b). Moreover, the F-P cavity along the thickness direction can only support single cavity mode, because the limited cavity length makes its mode spacing larger than the bandwidth of the optical gain. Therefore, single-mode lasing can be achieved when the cavity mode of the DBR cavity is carefully adjusted into the emission band of the CdS NRs. It should be noted that the emission light can also be guided freely at the NR length or width directions and reflected by the two opposite endfaces to realize the light gain and lasing, but it can only give multi-mode lasing, corresponding to intrinsic modes of F-P cavity at the length direction [21].

Figure $2 \mathrm{~b}$ shows the excitation fluence-dependent intensity for the emission at $\sim 520 \mathrm{~nm}$. The super linear increase of the PL intensity with the fluence indicates a lasing threshold of $\sim 8 \mu \mathrm{J} / \mathrm{cm}^{2}$, which is more than one order lower than those of the previously reported 
semiconductor NWs or NRs with naturally cleaved endfaces $\left(\sim 100 \mu \mathrm{J} / \mathrm{cm}^{2}\right)[35-36,47]$. The exhibited very low lasing threshold for the CdS NRs in the DBR cavity is contributed to the high reflectivity of both the bottom and the top DBRs, which increases the round-trip gain length through the repeated reflection and active transmission of the emitted light in the cavity (schematically shown in Figure 1b).

TRPL measurements were used to study the lasing mechanism of the CdS NRs in DBR cavity. Figure 3a and $b$ show the streak camera images of the sample excited at the power below $\left(5.0 \mu \mathrm{J} / \mathrm{cm}^{2}\right)$ and above $\left(14.3 \mu \mathrm{J} / \mathrm{cm}^{2}\right)$ the lasing threshold, respectively. A broad SPE emission band is observed with a lifetime of about several hundred picoseconds when the pump fluence is $5.0 \mu \mathrm{J} / \mathrm{cm}^{2}$. With increasing the pump fluence, the emission band is gradually narrowed accompanying the decrease of the lifetime, and a sharp emission line is finally observed with the lifetime of about ten picoseconds at the pump fluence of $14.3 \mu \mathrm{J} / \mathrm{cm}^{2}$. These clearly demonstrate the transition of the emission from SPE to stimulated emission. Figure $3 \mathrm{c}$ gives the decay profiles extracted from the streak camera images at the pump fluence of 5.0, 9.1 and $14.3 \mu \mathrm{J} / \mathrm{cm}^{2}$, respectively. At the fluence of $5.0 \mu \mathrm{J} / \mathrm{cm}^{2}$, the decay profile can be fitted by a monoexponential decay function with a time constant of $703.4 \mathrm{ps}$, which is related with the excitonic radiative recombination [48, 49]. Above the threshold, differently, the decay profiles can be fitted well by the biexponential decay functions, from which a fast $\left(\tau_{1}\right)$ and a slow $\left(\tau_{2}\right)$ lifetime were obtained, simultaneously. When the pump fluence is increased from 9.1 to $14.3 \mu \mathrm{J} / \mathrm{cm}^{2}, \tau_{1}$ decreases rapidly from 43.5 to $15.7 \mathrm{ps}$, while $\tau_{2}$ decreases slowly from 499.1 to 279.8 ps (see Supplementary Table S1). The rapid decrease of $\tau_{1}$ indicates the occurrence of the coherent recombination, which coincides with the electron-hole plasma (EHP) recombination at high pump fluence $[48,49]$. Additionally, with increasing the fluence, the proportion of the fast decay component in the total decay process increases from zero to 98.6\% (see Supplementary Table 1), which shows the EHP recombination dominates the 
carrier decay process at high pump levels, resulting in the almost complete transition from SPE to lasing [50].

Further analysis shows that the achieved single-mode lasers have a linear polarization with a very high polarization degree. Figures $4 \mathbf{a}$ and $\mathbf{b}$ are the polarization dependent emission spectra for the SPE and the lasing, respectively, when the electric field of emission light is separately parallel $\left(E_{e m} / / l\right.$, red curves) and perpendicular $\left(E_{e m} \perp l\right.$, black curves) to the length of the CdS NR (schematically shown in the inset). For the SPE, the emission intensity for $E_{\text {em }}$ $/ / l$ is stronger than that for $E_{e m} \perp l$, while for the lasing emission, the emission intensity for $E_{e m} \perp l$ is far stronger than that for $E_{e m} / / l$. The orthogonal polarization between the SPE and the lasing emission can be further confirmed by the polarization angle $\left(\varphi, 0^{\circ}\right.$ denotes $\left.E_{e m} / / l\right)$ dependent peak intensity plots in Figures 4c (SPE) and d (lasing). Experimental data (scatterplots) in both the plots can be fitted well by a function of $\cos ^{2} \varphi$ (solid lines) with the maximum intensity at $0^{\circ}$ and $90^{\circ}$ for SPE and lasing, respectively. The polarization degree, defined as $\mathrm{P}=\left(\mathrm{I}_{\max }-\mathrm{I}_{\min }\right) /\left(\mathrm{I}_{\max }+\mathrm{I}_{\min }\right)$, is determined from the measured data to be $40.5 \%$ and 97.4\% respectively for the SPE and the lasing. For wurzite structured CdS NRs with the size of both the length and the width larger than the exciting wavelength, the polarization of the SPE at room temperature is mainly determined by the selection rule of excitonic transitions including A- and B-excitons [51-53]. Because the $c$-axis of the CdS NRs is along their width direction $(w)[13,54]$, the PL spectrum obtained at $E_{\text {em }} / / l$ arises from both A and B-excitons, whereas the PL at $E_{e m} \perp l$ arises only from B-exciton [43, 51-53, 55]. The photon energy difference of the peak wavelength between the $E_{e m} / / l$ and $E_{e m} \perp l$ spectra is $\sim 19 \mathrm{meV}$, which agrees well with the energy separation between A- and B-excitons, $16 \mathrm{meV}$ [56]. This further confirms that the A- and B-exciton transition dominates the SPE at $E_{e m} / / l$ and $E_{e m} \perp l$, respectively. 
The polarization of the lasing emission is determined by the modes coupled to the lasing cavity [57]. Figure $4 \mathrm{e}$ is the electric field intensity distribution simulation of the device crosssection for the fundamental mode at $520 \mathrm{~nm}$ using finite-element method, where the parameter of the model is set as the same as that of the designed DBR. Clearly, the polarization of electric field at fundamental mode is dominant in the width $(x)$ direction with a very weak component in the length $(y)$ and thickness $(z)$ direction, which agrees well with the experimental results. Additionally, the cavity field is observed to be mainly confined in the NR region, which may decrease the round-trip loss and hence the lasing threshold. Again, the results reveal that the single-mode lasing comes from the coupling of CdS NRs with the dielectric DBR cavity.

The resonance wavelength $(\lambda)$ of the CdS embedded DBR F-P cavity can be expressed as: $\lambda=\frac{2\left(n_{S} d_{S}+n_{C d S} d_{C d S}\right)}{m}$, where $n_{S} d_{S}$ is the optical length of $\mathrm{SiO}_{2}$ cavity $\left(\lambda_{0} / 2=260 \mathrm{~nm}\right), \mathrm{n}_{\mathrm{CdS}}$ is the refractivity index of $\mathrm{CdS}(2.5)$, and $m$ is an integers, denoting the order of the cavity mode [42]. Based on this relation, $\lambda$ can be decided by the thickness of the examined CdS NR $\left(\mathrm{d}_{\mathrm{CdS}}\right)$. Figure 5 gives the obtained lasing spectra of the CdS ribbons with the thickness $\left(\mathrm{d}_{\mathrm{CdS}}\right)$ of 400 (a), 230 (b), 304 (c), 110 (d), and $316 \mathrm{~nm}$ (e), respectively embedded in the same DBR cavity (the thickness measurements are given in Supporting Information, Figure S3). The result shows that the wavelength of the laser mode can be modulated continuously from $\sim 498$ to $\sim 520 \mathrm{~nm}$, covering the whole band-edge emission band of CdS, which is well consistent with the calculation using the above relation. Based on the calculation, the order of the lasing mode are $5,3,4,2$, and 4 , for the ribbon with the thickness of 400, 230, 304, 110, and 316, respectively. Further improvements can be obtained with combinatorial etching technique, or position-related ribbon thickness control techniques, by which different resonance wavelength F-P DBR cavities can even be integrated on a single NR [42, 58, 59]. 


\section{Conclusion}

In summary, CdS NRs were embedded in dielectric DBRs microcavity easily. Single-mode lasers were obtained with low threshold under femtosecond laser excitation, attributed to the high reflectance dielectric DBRs which can significantly increase the round-trip gain for the cavity along the thickness direction of the ribbons. The TRPL results reveal that the lasing action is an EHP recombination process. The emission polarization and numerical simulation studies demonstrate that the single-mode lasing has high polarization degree of $97.4 \%$, which originates from the coupling of CdS NRs with the cavity resonance mode. The wavelength of the lasing mode can be tuned at the whole bandwidth of $\mathrm{CdS}$ emission by varying the length of the cavity. The wavelength of single-mode lasers can be easily extended from UV to IR using the semiconductor NRs with the corresponding bandgaps combining with the proper designed DBRs, which are expected to be applied in the on-chip communication and signal processing, display, and so on.

\section{Acknowledgements}

The authors are grateful to the NSF of China (Nos. 51525202, 61574054 and 61474040), the National Basic Research Program of China (No. 2012CB932703), Youth Innovation Promotion Association CAS (2012189), the Shanghai Science and Technology Foundations (15DZ2282100, 16DZ2290600).

\section{References}

[1] R. Yan, D. Gargas, P. Yang, Nat. Photonics 3 (2009) 569-576.

[2] K. J. Vahala, Nature 424 (2003) 839-846.

[3] Y. Li, F. Qian, J. Xiang, CM. Lieber, Mater. Today 9 (2006) 18-27.

[4] R. Agarwal, C. J. Barrelet, CM. Lieber, Nano Lett. 5 (2005) 917-920. 
[5] X. Tang, Z. Hu, W. Chen, X. Xing, W.Hu, J. Qiu, Z. Zang, J. Du, Y. Leng, X. Jiang, L. Mai, Nano Energy, in press (http://dx.doi.org/10.1016/j.nanoen.2016.08.062)

[6] R. Agarwal, Small 4 (2008) 1872-1893.

[7] J. C. Johnson, H. Q. Yan, R. D. Schaller, L. H. Haber, R. J. Saykally, P. Yang, J. Phys. Chem. B 105 (2001) 11387-11390.

[8] H. Yan, H. S. Choe, S. W. Nam, Y. J. Hu, S. Das, J. F. Klemic, J. C. Ellenbogen, C. M. Lieber, Nature 470 (2011) 240-244.

[9] X. Liu, P. Xu, Y. Wu, Z. Yang, C. Meng, W. Yang, J. Li, D. Wang, X. Liu, Q. Yang, Nano Energy14 (2015) 340-354.

[10] R. S. Friedman, M. C. McAlpine, D. S. Ricketts, D. Ham, C. M. Lieber, Nature 434 (2005) 1085.

[11] D. J Sirbuly, M. Law, P. Pauzauskie, H. Q. Yan, A. V. Maslov, K. Knutsen, C. Z Ning, R. J. Saykally, P. Yang, Proc. Nat. Acad. Sci. U.S.A. 102 (2005) 7800-1805.

[12] A. L. Pan, W. C. Zhou, E. S. P. Leong, R. B. Liu, A. H. Chin, B. S. Zou, C. Z. Ning, Nano Lett. 9 (2009) 784-788.

[13] A. L. Pan, R. B. Liu, B. S. Zou, Appl. Phys. Lett. 88 (2006) 173102.

[14] A. V. Maslov, C. Z. Ning, Appl. Phys. Lett. 83 (2003) 1237-1239.

[15] X. Wang, X. Zhuang, S. Yang, Y. Chen, Q. Zhang, X. Zhu, H. Zhou, P. Guo, J. Liang, Y. Huang, A. Pan, X. Duan, Phys. Rev. Lett. 115 (2015) 027403.

[16] M. A. Zimmler, F. Capasso, S. Mueller, C. Ronning, Semicond. Sci. Technol. 25 (2010) 024001.

[17] S. S. Wang, Z. F. Hu, H. K. Yu, W. Fang, M. Qiu, L. M. Tong, Opt. Express 17 (2009) $10881-10886$.

[18] R. F. Oulton, V. J. Sorger, T. Zentgraf, R. Ma, C. Gladden, L. Dai, G. Bartal, X. Zhang, Nature 461 (2009) 629-632. 
[19] Q. Zhang, G. Li, X. Liu, F. Qian, Y. Li, T. C. Sum, C. M. Lieber, Q. Xiong, Nat. Comm. 5 (2014) 4953.

[20] Y. Yin, T. Qiu, J. Li, P. K. Chu, Nano Energy 1 (2012) 25-41.

[21] A. Das, J. Heo, J M. ankowski, W. Guo, L. Zhang, H. Deng, P. Bhattacharya, Phys. Rev. Lett. 107 (2011) 066405.

[22] P. Guo, X. Zhuang, J. Xu, Q. Zhang, W. Hu, X. Zhu, X. Wang, Q. Wan, P. He, H. Zhou, A. Pan, Nano Lett. 13 (2013) 1251-1256.

[23] S. Gradečak, F. Qian, Y. Li, H. G. Park, C. M. Lieber, Appl. Phys. Lett. 87 (2005) 173111

[24] M. A. Zimmler, J. Bao, F. Capasso, S. Müller, C. Ronning, Appl. Phys. Lett. 93 (2008) 051101

[25] B. E. A. Saleh, M. C. Teich, Fundamentals of Photonics. (New York: Wiley Interscience, 2007).

[26] D. Botez, Solid State Electron Dev. IEE Proc. 129 (1982) 237-251.

[27] A. Javan, W. R. Bennett, Jr., D. R. Herriott, Phys. Rev. Lett. 6 (1961) 106-110.

[28] W. H. Loh, B. N. Samson, L. Dong, G. J. Cowle, K. Hsu, J. Lightwave Technol. 16 (2003) 114-118.

[29] J. J. Zayhowski, A. Mooradian, Opt. Lett. 14 (1989) 24-26.

[30] J. Li, C. Meng, Y. Liu, X. Wu, Y. Lu, Y. Ye, L. Dai, L. Tong, X. Liu, Q. Yang, Adv. Mater. 25 (2013) 833-837.

[31] S. H. Park, S. L. Chuang, Appl. Phys. Lett. 72 (1998) 287.

[32] A. Yamamoto, T. Kido, T. Goto, Y., Chen T. Yao, A. Kasuya, Appl. Phys. Lett. 75 (1999) 469-471.

[33] P. J. Pauzauskie, D. J. Sirbuly, P. Yang, Physic. Rev. Lett. 96, 143903 (2006). 
[34] R. Ma, X. Wei, L. Dai, S. Liu, T. Chen,S. Yue, Z. Li, Q. Chen, G. G. Qin, Nano Lett. 9 (2009) 2697-2703.

[35] Y. Xiao, C. Meng, P. Wang, Y. Ye, H. Yu, S. Wang, F. Gu, L. Dai, L. Tong, Nano Lett. $11(2011)$ 1122-1126.

[36] Y. Xiao, C. Meng, X. Wu, L. Tong, Appl. Phys. Lett. 99, 023109 (2011).

[37] H. Gao, A. Fu, S. C. Andrews, P. Yang, Proc. Nat. Acad. Sci. U.S.A. 110 (2013) 865869.

[38] L. Chen, E. Towe, Appl. Phys. Lett. 89 (2006) 053125.

[39] A. Fu, H. Gao, P. Petrov, P. Yang, Nano Lett. 15 (2015) 6909-6913.

[40] C. J. Barrelet, J. Bao, M. Lončar, H. Park, F. Capasso, C. M. Lieber, Nano Lett. 6 (2006) $11-15$.

[41] A. Pan, D. Liu, R. Liu, F. Wang, X. Zhu, B. S. Zou, Small 1 (2005) 980-983.

[42] S. Wang, X. Chen, W. Lu, L. Wang, Y. Wu, Z. Wang, Opt. Lett. 31 (2006) 332-334.

[43] L. X. Sun, D. H. Kim, K. H. Oh, R. Agarwal, Nano Lett. 13 (2013) 3836-3842.

[44] J. Xu, L. Ma, P. Guo, X. Zhuang, X. Zhu, W. Hu, X. Duan, A. Pan, J. Am. Chem. Soc. 134 (2012) 12394-12397.

[45] J. A. Zapien, Y. Jiang, X. M. Meng, W. Chen, F. C. K. Au, Y. Lifshitz, S. T. Lee, Appl. Phys. Lett. 84 (2004) 1189-1191.

[46] Y. Ma, X. Guo, X. Wu, L. Dai, L. Tong, Adv. Opt. Photonics 5 (2013) 216-273.

[47] S. Geburt, A. Thielmann, R. Röder, C. Borschel, A. McDonnell, M. Kozlik, J. Kühnel, K. A. Sunter, F. Capasson, C. Ronning, Nanotechnology 23 (2012) 365204.

[48] R. B. Liu, J. H. Cao, Z. A. Li, Q. Wang, Q. L. Zhang, P. B. He, B. S. Zou, A. L. Pan, J. Phys.: Condens Matter 21 (2009) 375302.

[49] J. C. Johnson, K. P. Knutsen, H. Yan, M. Law, Y. Zhang, P. Yang, R. J. Saykally, Nano Lett. 4 (2004) 197-204. 
[50] A. L. Pan, R. B. Liu, Q. Yang, Y. C. Zhu, G. Z. Yang, B. S. Zou, K. Q. Chen, J. Phys. Chem. B 109 (2005) 24268-24272.

[51] D. G. Thomas, J. J. Hopfield, Phys. Rev. 116 (1959) 573-582.

[52] R. Venugopal, P. I. Lin, C. C. Liu, Y. T. Chen, J. Am. Chem. Soc. 127 (2005) $11262-$ 11268.

[53] C. X. Shan, Z. Liu, S. K. Hark, Phys. Rev. B 74 (2006) 153402.

[54] L. Dong, J. Jiao, M. Coulter, L. Love, Chem. Phys. Lett. 376 (2003) 653-658.

[55] L. X. Sun, M. L. Ren, W. J. Liu, R. Agarwal, Nano Lett. 14 (2014) 6564-6571.

[56] L. C. Lew Yan Voon, M. Willatzen, M. Cardona, N. E. Christensen, Phys. Rev. B 53 (1996) 10703-10714.

[57] H. Y. Li, S. Rühle, R. Khedoe, A. F. Koenderink, D. Vanmaekelbergh, Nano Lett. 9 (2009) 3515-3520.

[58] S. Wang, D. Liu, B. Lin, X. Chen, W. Lu, Appl. Phys. B 82 (2006) 637-641.

[59] S. Wang, M. Li, C. Xia, H. Wang, X. Chen, W. Lu, Appl. Phys. B 88 (2007) 281-284. 
Figure1. (a) The side view schematic of the single-mode laser device formed with a CdS NR sandwiched between two dielectric DBRs. (b) Amplified view of interface between CdS NR and DBRs, arrows denotes the optical path in CdS and DBRs, respectively. (c) SEM of the representative CdS NRs embedded in the DBR cavity, at the viewing angle of $45^{\circ}$. Inset: the enlarged image of the solid-line marked region. (d) The transmission spectrum of the one DBR (upper panel) and the PL emission of CdS without the microcavity (lower panel). Inset: the $\mu-\mathrm{R}$ spectra of the representative CdS NR sandwiched in the DBRs.
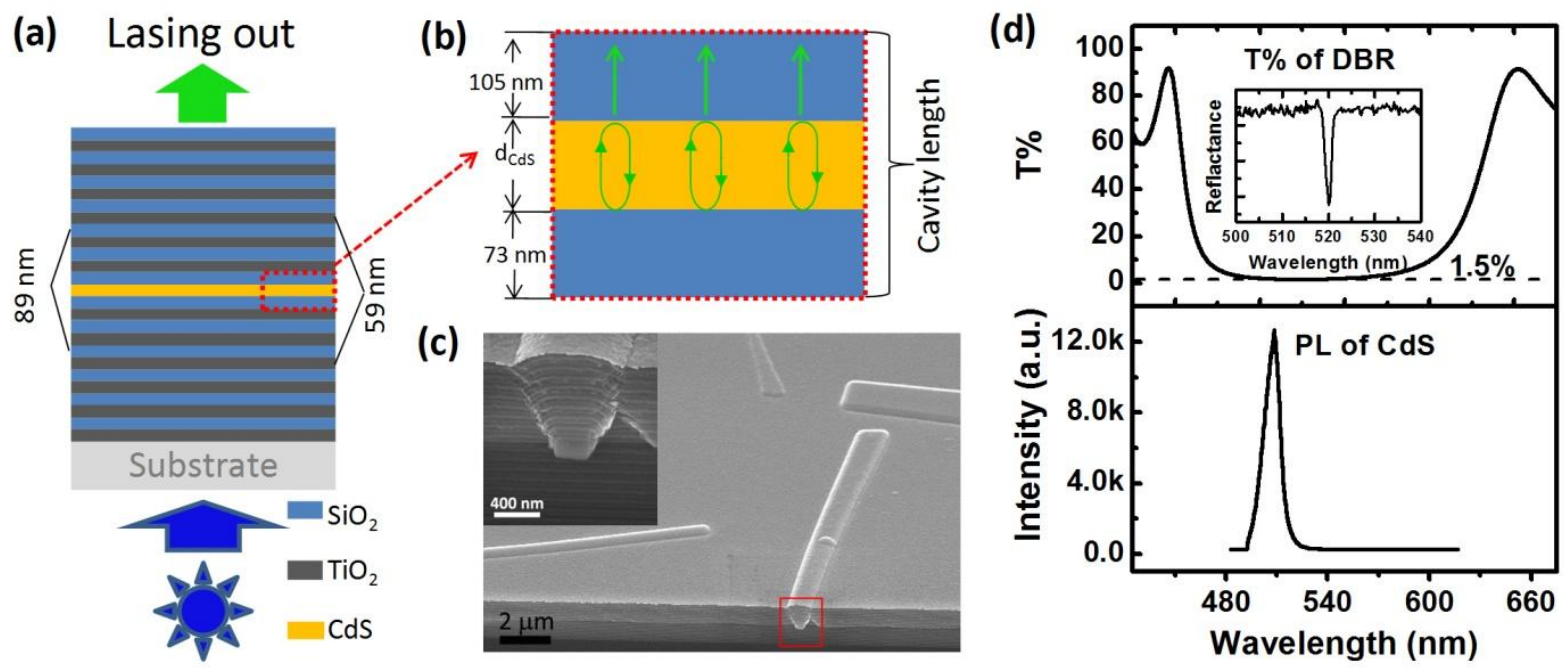
Figure 2. (a) The pump fluence dependent emission spectra of a CdS NR in the DBR microcavity. Inset: The real color micro image for the device illuminated from the bottom substrate side with the pump fluence above the threshold. Scale bar is $10 \mu \mathrm{m}$. (b) Emission intensity versus the pump fluence of the pumped device. The emission intensity is the peak intensity of the single-mode lasing at $520 \mathrm{~nm}$.
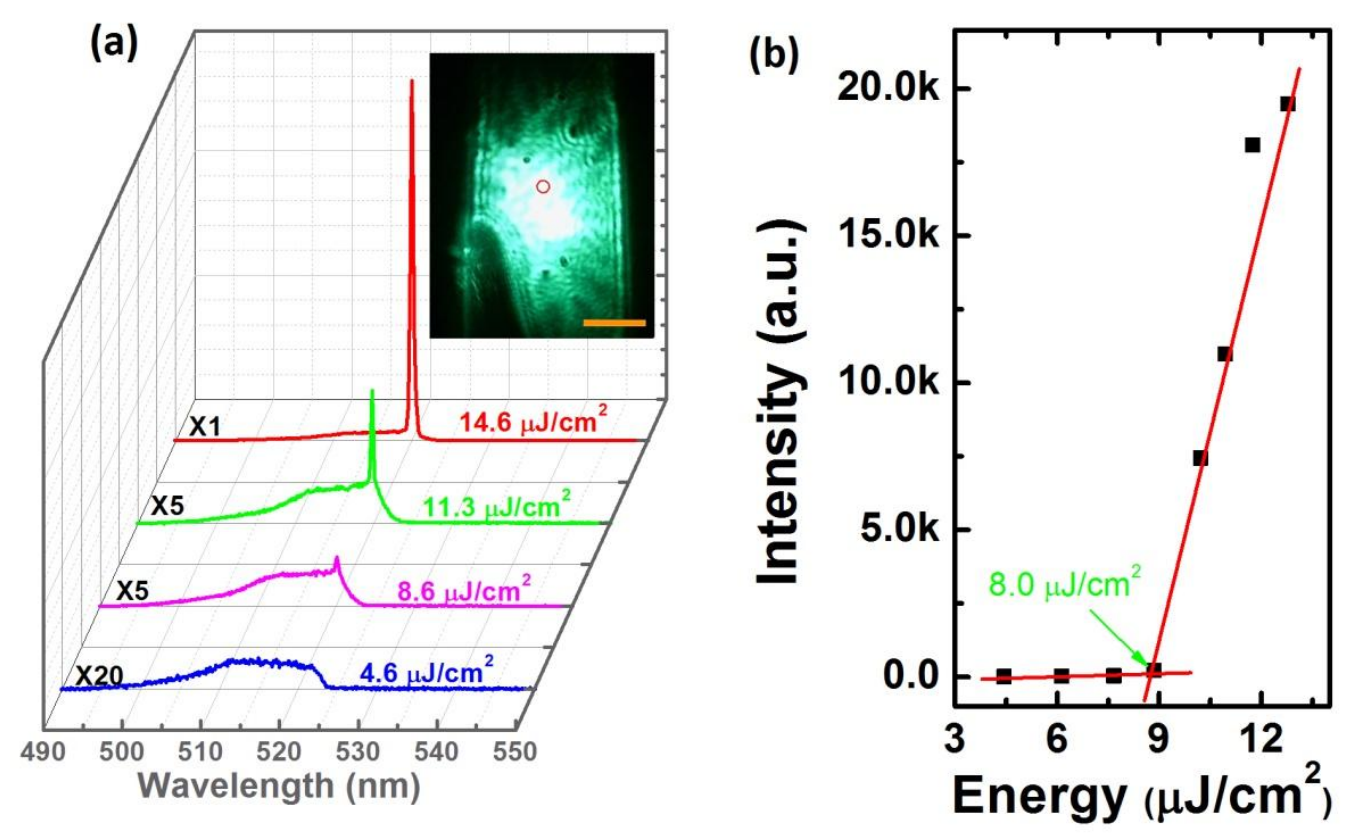
Figure 3. The streak camera image of the device excited below (a) and above (b) the lasing threshold. (c) The decay profile of the emission measured under the pump of 5.0, 9.1 and 14.3 $\mu \mathrm{J} / \mathrm{cm}^{2}$, respectively. Solid lines are the fitted results.
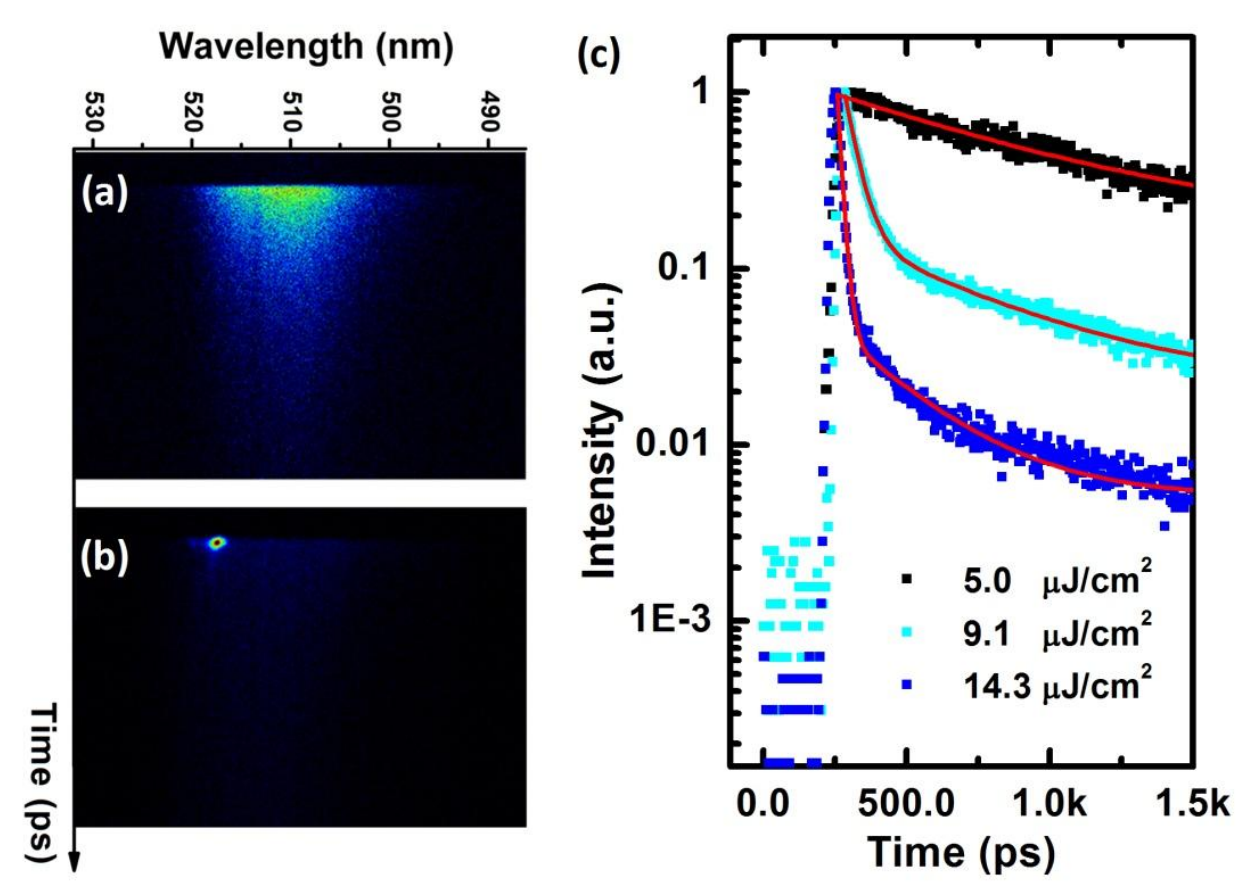
Figure 4 The SPE (a) and lasing emission (b) spectra of the device for emission light electric field parallel (red line) and perpendicular (black line) to the length $(l)$ of the CdS NR. Inset, the relation between the emission light electric field direction and the length $(l)$ or width $(w)$ of the NR. (c) and (d) The polar plots of peak intensity versus detection polarization angle $(\varphi)$ for SPE and lasing, respectively. (e) The simulated electric field distribution of a device from the cross-sectional view. The size of the cavity is $1.2 \times 0.4 \mu \mathrm{m}$, and the core CdS NR is $1.0 \mu \mathrm{m}$ (width) $\times 0.2 \mu \mathrm{m}$ (thickness).
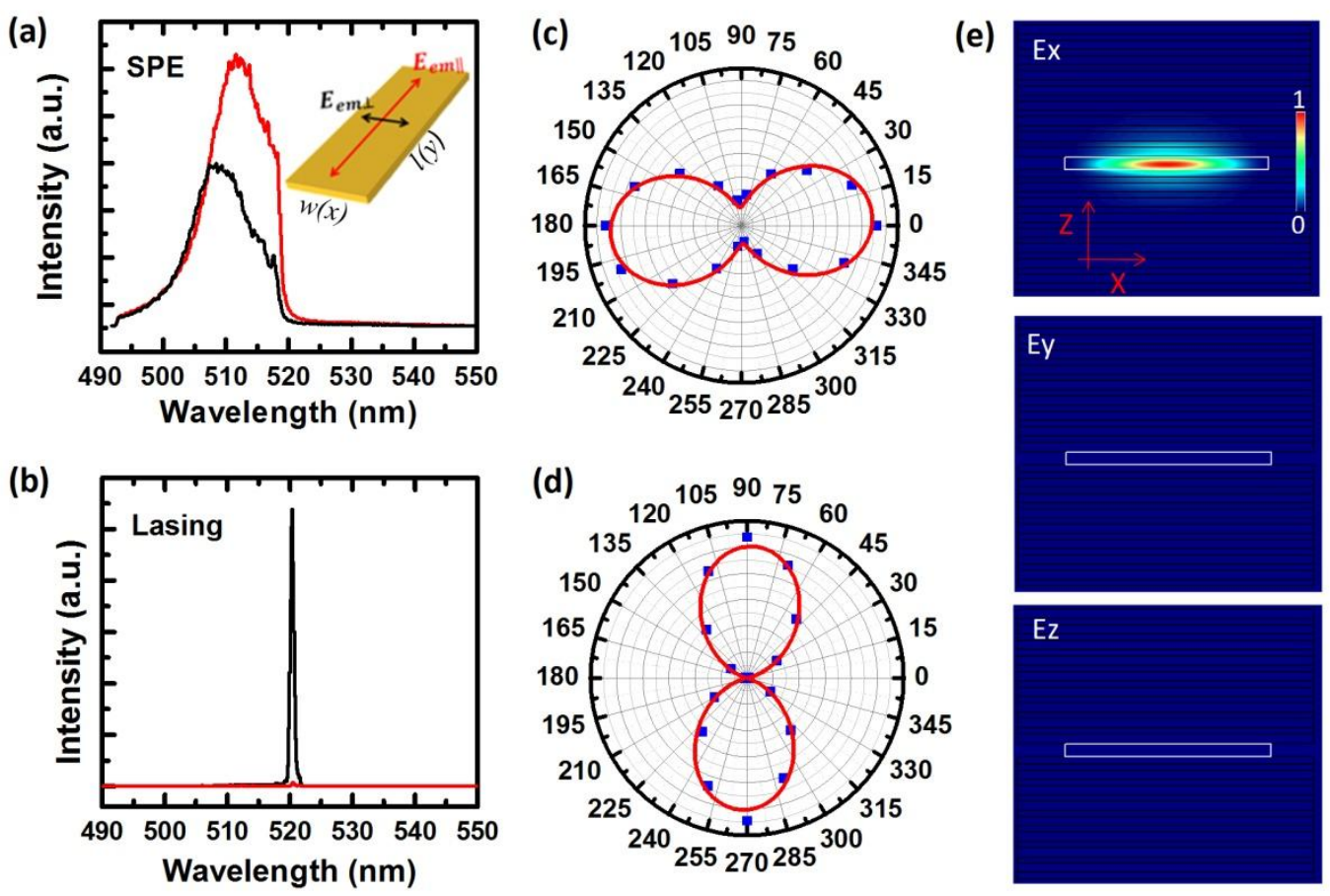
Figure 5. The lasing spectra of CdS NRs embedded in DBR microcavity with the thickness of 400 (a), 230 (b), 304 (c), 110 (d) and 316 nm (e).

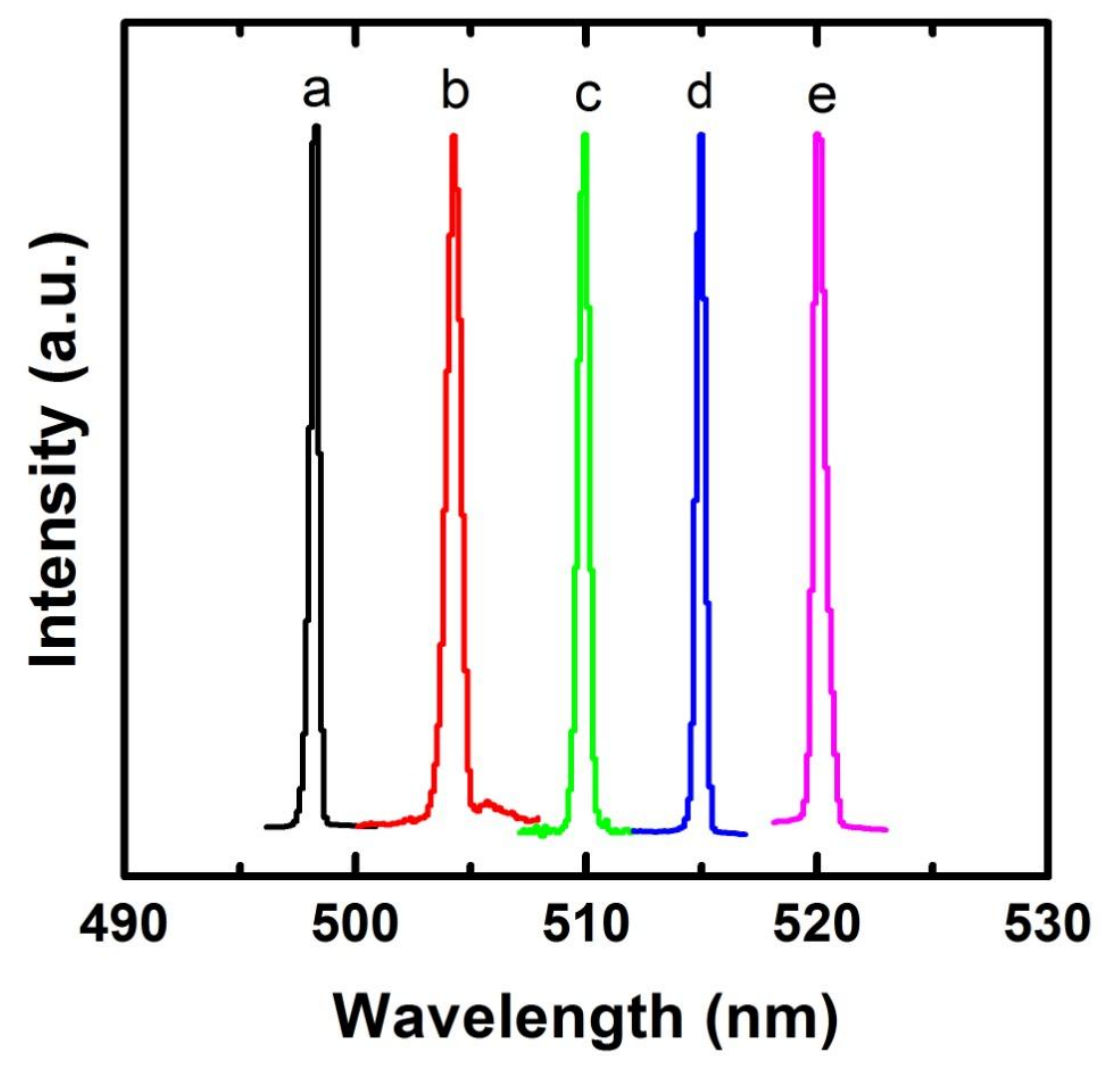



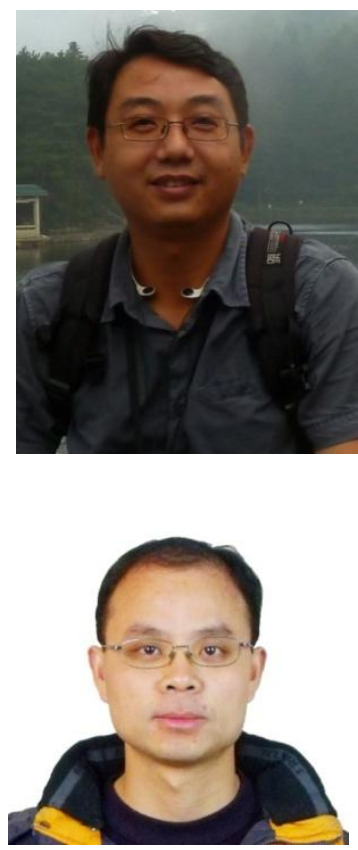

Qinglin Zhang received the B. S. and $\mathrm{Ph}$. D. from the College of Chemistry of Jilin University in 2001 and 2006, respectively. He is currently an Associate Professor in School of Physics and Electronics of Hunan University. He has published over 20 research papers and authorized one China patent. His research interests mainly concerns the fabrication, optical characterization and applications of semiconductor nanostrucutres, and the development of new experimental techniques to study the novel optical and optoelectronic properties of nanostructured semiconductor materials.

Shao-Wei Wang received his Ph.D. (2003) degree in microelectronics and solid state electronics from Shanghai Institute of Technical Physics, Chinese Academy of Sciences, China. He is a professor of the institute and works at National Laboratory for Infrared Physics. His research interests include artificial photonic structure and devices, such as interaction between high-Q optical cavity and low-dimensional materials, integrated-cavities for micro-spectrometers, solar selective absorbers, metamaterial polarizers, and optical thin films. He has published more than 50 research papers and authorized one US patent. He got LU JIAXI Young talent award (2009), RAO YUTAI basic optical award (2007) , National Natural science award $\left(2014,4^{\text {th }}\right.$ principal achiever), National Technological Invention Award (2011, $5^{\text {th }}$ principal achiever), Shanghai Technological Invention Award (2010, $7^{\text {th }}$ principal achiever), Shanghai Natural science award $\left(2007,5^{\text {th }}\right.$ principal achiever $)$, etc.

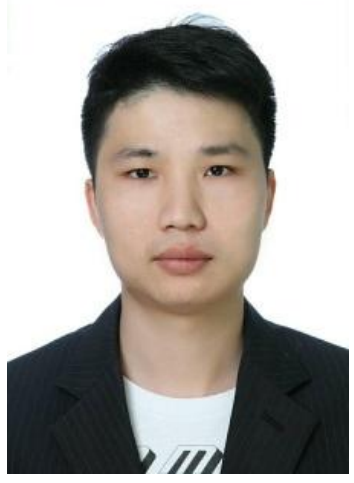

Xingxing Liu received his Bachelor's Degree (2011) degree in optoelectronic technology science from Donghua University, China. He is a Ph.D. candidate of microelectronics and solid state electronics from Shanghai Institute of Technical Physics, Chinese Academy of Sciences, China. His research interests include artificial photonic structure and devices, such as interaction between high-Q optical cavity and lowdimensional materials, integrated-cavities for micro-spectrometers and optical thin films. He has published over 10 papers. He got University of Chinese Academy Sciences Merit Student award $(2015,2016)$ and Donghua University Merit Student award (2010), etc.

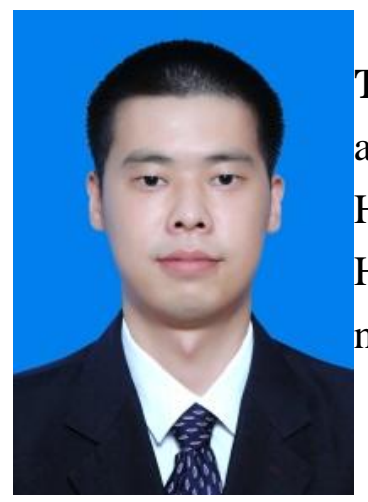

Tianren Chen received the B.S. degree from the Department of Physics and Electronics of Hunan Institute of Science and Technologyin 2014. $\mathrm{He}$ is now a master candidate at School of Physics and Electronics of Hunan University. His research interests mainly focus on synthesis of novel II-VI semiconductor nanostructures for optical applications. 


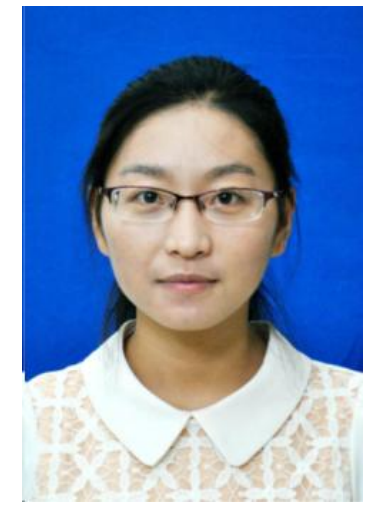

Huafen Li received her Bachelor's degree (2015) in mathematics and applied mathematics from the Ocean University of China. She now works as a junior engineer at National Laboratory for Infrared Physics, Shanghai Institute of Technical Physics. She is familiar at optical thin films and optical coating systems. She published 2 papers and applied 1 patent.
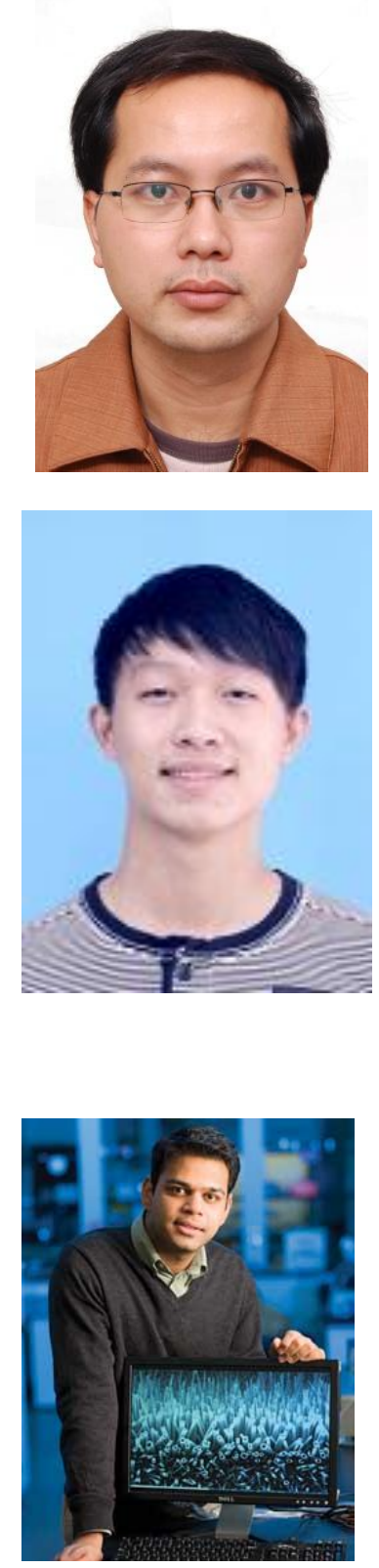

Ritesh Agarwal earned his undergraduate degree from the Indian Institute of Technology, Kanpur in 1996, and a master's degree in chemistry from the University of Chicago. He received his PhD from the University of California at Berkeley in 2001. Afterwards, he was a postdoctoral fellow at Harvard University. He is currently a professor in the Department of Materials Science and Engineering at the University of Pennsylvania. His research interests include understanding and engineering light-matter interactions at the nanoscale using a variety of one- and two-dimensional semiconductors, plasmonic nanocavities, and studying phase transitions and electronic memory switching at the nanoscale. He has published over 64 peer-reviewed papers. 


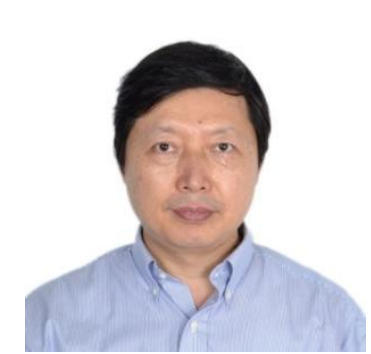

Wei Lu received his Ph.D. (1988) degree in Material and Device from Shanghai Institute of Technical Physics, Chinese Academy of Sciences, China. He is the director (2013-) and professor (1993-) of the institute. He is associated editor of $J$ Appl. Phys. beginning from 2010 . He is the member of academy committee of five State Key Labs in China and program member of six international conferences. His areas of specialty are infrared physics, opto-electronics, semiconductor material and devices, condensed matter material. He has published more than 150 papers and authorized one US patent. He got eleven scientific awards in China including first principal achiever of National Natural science award (2014), National Technological Invention Award (2011), Shanghai Technological Invention Award (2010), Shanghai Natural science award (2007), etc.

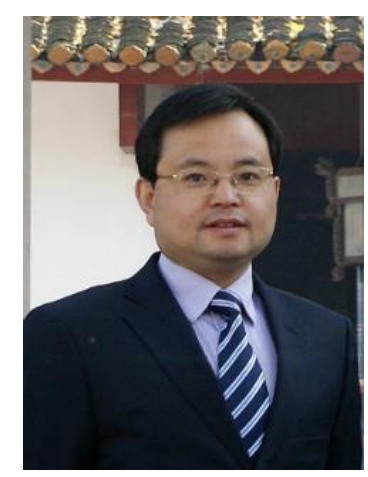

Anlian Pan received his $\mathrm{PhD}$ from the Institute of Physics, Chinese Academy of Sciences in 2006. Afterwards, he worked one year as a Humboldt Research Fellow with Prof. Ulrich Gosele at the Max Planck Institute of Microstructure Physics, and then joined Arizona State University as a Postdoctoral Fellow, where he became a Research Assistant Professor. He came back to Hunan University and worked as the distinguished professor of "Furong" scholar in Hunan province in 2010. He has published more than 140 papers. His research interests include the micro-nano optical, electronics of semiconductor nanostructructures. 


\section{Low Threshold, Single-mode Laser Based on Individual CdS Nanoribbons in Dielectric DBR Microcavity}

\section{Graphical Abstract}

It is reported that single-mode, low threshold nanoscale lasers based on CdS nanoribbons sandwiched between two dielectric distributed Bragg reflectors (DBRs). Under optical pumping, the band edge emission of embedded $\mathrm{CdS}$ NR can be confined in the DBR microcavity due to the high reflectivity of the DBRs, which results in a low threshold lasing with single mode.
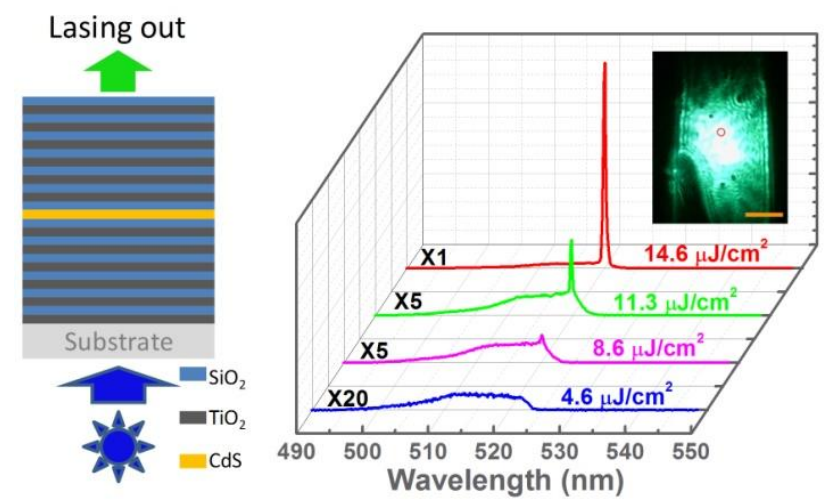